\title{
DISCUSSION (Artru and Lanz)
}

MICHAUD: Are the theoretical calculations sufficiently accurate to predict if the absorption is strong enough to explain the strengths of the features, if not their positions?

ARTRU: Yes, the theoretical values of autoionization widths and oscillator strengths generally give the right order of magnitude for the width and strength, but we can not predict precisely the wavelength of the features.

MÉGESSIER: Did you try to compute synthetic spectra including all the elements expected to be overabundant, in order to estimate the amplitude of the depression which can not be explained by the overabundance of the element itself?

ARTRU: We only did the calculation for Si II, but we could introduce other species (Si III, Fe II, for example). These will not fill the gap. Contributions of unknown lines, especially autoionization lines, must be important.

HUBENÝ: Could you, for the average user of the data, please comment on the precision of the data from the paper by Artru, Jamar, Petrini \& Praderie (Astron. Astrophys., 99, p. 401, 1981)? I am a little surprised that you have not included all of these results in the calculations here. To what extent can one trust this data, particularly for autoionization lines, when calculating synthetic spectra?

ARTRU: Yes, this paper looks like a step backward from the 181 paper, but in the earlier work we put in the synthesis all the data predicted in the calculation, including the autoionization widths. However, at that time we did not compare to IUE spectra, so we did not try to fit the predicted lines to the observed ones. Later, we tried to do this, and found that it is not trivial to assign each observed line to a predicted one, and vice versa. We think the predicted spectrum should be globally correct, especially the autoionization widths, but the wavelengths derived from differences of theoretical levels are not accurate enough for identification of individual lines.

COWLEY: A number of years ago, the Si star HD 34452 was studied by Tomley, Wallerste in \& Wolff (Astron. Astrophys., 9, p. 380, 1970), and they found a result which was quite remarkable for Si stars, that the oxygen was apparently markedly overabundant. This interesting result should be confirmed and followed up, because oxygen is usually thought to be underabundant in magnetic Ap stars. My question is, do you think $\mathrm{Si}$ is capable of explaining all of the large absorption features, if the autoionization levels have the most favourable properties, or do you think that you must include absorption from other ions such as $\mathrm{Fe}$, or if 0 is overabundant, possibly from that ion?

ARTRU: I think the Si II can explain the width we observe for, as an example, the 1400 A depression. Unfortunately, we can not yet prove that the right line is located there. To do this, we need additional laboratory data.

ADELMN: I have a comment on the previous question asked by Cowley. In normal B and A stars, one observes only a few moderate strength lines of 0 I in IUE spectra in the region 1300 - $1360 \AA$. Changing the oxygen abundance will not affect the total amount of line absorption very much. 
There are no observed $O$ II lines in the wavelengths covered by IUE spectra.

ARTRU: Also, chlorine is said to be overabundant in this star, but I did not see evidence of excess $\mathrm{Cl}$ lines in the range of wavelengths $I$ studied.

\section{DISCUSSION (Leckrone)}

WEISS: Can you please comment on your experience with co-adding IUE spectra. In particular, how does the signal-to-noise ratio improve?

ADELMAN: Leckrone and I have studied the noise of high-resolution IUE spectra by comparing individual exposures with our co-additions. For properly exposed regions, the fixed-pattern noise is $6 \%$ of the total signal, while random noise is $4 \%$ of the total signal. The fixed-pattern noise, which is an instrumental effect variable over a time of order one year, can be shifted relative to the spectrum by $\pm 25 \mathrm{~km} / \mathrm{s}$, by moving the star relative to the center of the large aperture. If one takes three exposures with the same exposure time, one with the star at the center, one at the left side, and the other at the right side, and then adds them with the stellar features in coincidence, then one can reduce the fixedpattern noise by a factor of $\sqrt{3}$. A similar factor applies to the reduction in random noise. The detailed causes of the fixed-pattern noise are not understood very well.

MICHAUD: In the fit to the 1942 \& $\mathrm{Hg}$ II line, how many parameters were allowed to vary, and to what extent were the abundances known when the gf-values were allowed to vary?

ADELMAN: The abundances of blending species are known from the best available optical analyses, as are the stellar parameters: effective temperature, surface gravity, microturbulence, and $v$ sin i. These are verified by examination of the ultraviolet data. The gf-values from the Kurucz and Peytremann list are used unless better ones are available. If necessary, and with some reluctance, the gf-values are adjusted to reproduce the observed region as well as possible in all the programme stars simultaneously. Essentially, we assume that we know gf times abundance, and we have to decouple them somehow.

DWORETSKY: The normal comparison star $\xi$ Oct is probably an unfamiliar choice to most people at this colloquium. It is fairly bright and near the South.Celestial Pole. Preliminary work on the IUE spectra by J.M. Jacobs at University College London showed that it appears to have nearly solar abundances of metals. Observations to obtain an accurate $v$ sin $i$. are now being made at ESO (by Iye of Japan). It looks normal and very sharp-lined on my $15 \mathrm{~A} / \mathrm{mm}$ spectra. As we all know, whenever we find a sharp-lined late B star it usually turns out to be peculiar, but I think this will turn out to be a good comparison star with essentially solar abundances. Unfortunately it can not be observed from the Crimean Astrophysical Observatory!

HUBENÝ: I have two questions. First, how is the opacity in the additional fictitious lines treated? I mean, for example, how are the lower excitation potential, species, gf-value, etc chosen?

ADELMAN: The fictitious line was selected to fill in properly the missing opacity for all stars. Leckrone selected $T i$ II, as its 
abundance is fairly constant from star to star. The excitation potential chosen was a typical one for a line of this species.

HUBENÝ: So the choice is by trial and error? For many stars?

ADELMAN: Yes. For example, Leckrone can be sure that it isn't a Mn II line, because it does not vary in strength from star to star like those lines.

HUBENÝ: Second, why was $\alpha$ Lyrae (Vega) not chosen as a standard comparison star for Space Telescope?

ADELMAN: Vega is not as sharp-lined as the standards chosen by Leckrone. There are suggestions in the literature concerning its possible variability. Most of these, however, can not be taken seriously.

DWORETSKY: I will discuss Vega on Friday, in my review paper.

ADELMAN: The entire HRS team has chosen to observe Sirius rather than Vega. To observe the entire spectrum at the highest resolution will take 1.8 hours. If no one has reserved Vega, I am sure that there will be a group which will propose to observe it, perhaps in the $R=25,000$ mode. The GTO programmes will be published in advance.

SEVERNY: Could you explain how you fixed the position of the continuum for the spectra?

ADELMAN: This is one of the most difficult and nasty problems one must handle. There are several approaches possible. In the 'heuristic' approach, I look at spectra of several stars at once and search for high points present in all the stars. I then assume that these define the continuum. This can be carried one step further. You compute synthetic spectra for these regions using the Kurucz and Peytremann line list, and study how abundance changes and rotation modify the appearance of these regions. This is the method I am actually trying to use, but I am not completely satisfied with it. Locating the continuum is one of the nastiest problems we have!

SCHÖNICH: What do you know about proposed observations of magnetic $\mathrm{CP}$ stars on the Space Telescope?

ADELMAN: Dr. Leckrone chose to observe $\mathrm{Hg}-\mathrm{Mn}$ stars and normal stars with his time as a Guaranteed Time Observer on the Hubble Space Telescope. I have no knowledge of planned observations of magnetic CP stars by any GTOs. 